\title{
Treatment of children's shaft fracture of tibia and fibula with ESIN fixation
}

\author{
Ping Liu, Zuo Wei, You-Xiu Wei, Wen-Xiao Sun, He-Wei Li, Song Huang, Wei Zou
}

Department of Orthopaedics, LiYuan Hospital, TongJi Medical College, HuaZhong University of Science and Technology, Wuhan, China.

E-mail: yanzi791005@163.com

Received 13 April 2011; revised 9 May 2011; accepted 2 June 2011.

\begin{abstract}
The objective is to evaluate Elastic Stable Intramedullary Nailing (ESIN) fixation through the treatment of children's shaft fracture of tibia and fibula. From January 2007 to January 2009, 16 cases of children's shaft fracture of tibia and fibula had been treated by ESIN. 15 cases were followed up after the surgery and the follow-up time was 4 - 12 months (4.5 in average). The results turn out that all the patients' fractures have got healed by Phase $I$ and the healing time is $\mathbf{1 0}$ weeks in average. The near joints' activity and affected extremity completely return to normal. This group of cases doesn't show any sign of infection, bone nonunion, malunion or epiphysis injury. And six patients have had their intramedullary nails taken out after six months. It can be concluded that ESIN is good for treating children's long diaphysis fracture which has difficulty in closed diaplasis and is suitable for children aged 6 - 13. ESIN has the advantages of causing small incision, slight injury of soft tissue, short surgery time, rapid recovery and few complications.
\end{abstract}

Keywords: Children; Shaft Fracture of Tibia and Fibula; ESIN; Intramedullary

\section{INTRODUCTION}

The traditional therapy for children's long diaphysis fracture is manual diaplasis or traction diaplasis performed with gypsum fixation or splinting which have the disadvantages of unsatisfied diaplasis and easy retransposition. In the last two decades, especially in the recent 10 years, with the appearance of new fixing materials and the increasing of people's requirements of life quality, more and more orthopedists have begun to use surgeries such as internal fixation with bone plate, intramedullary nail fix- ation and external fixation, etc., for such sort of fracture. But these methods may produce com- plications such as anomalies of skeletal growth and nail infection, which will limit the application in treating children's long diaphsis fracture. ESIN, which can be also called Titanic Elastic nailing (TEN), is a kind of internal fixation devices made of titanium alloy with sickle shape elbow and is specially used to treat children's long disphysis fracture. We had used TEN for operations in various types of children's fracture of tibia and fibula from January of 2007 to October of 2008 and the summary is as below.

\section{MATERIALS AND METHODS}

\subsection{General Data}

Among these 16 cases, 10 are male patients and the other 6 are female, aged from 7.3 to 15 years old (10.2 in average); 10 cases belong to closed fracture and 1 open fracture. And their bodies have varying degrees of angular deformity in hospital. Taking X-ray photographs and measuring the size of the medullary cavity before the surgery were taken as the basis of choosing the diameter of the TEN (Figures 1(a) and (b)).

\subsection{Treatments}

The patient laid his back on the light operation bed under general anesthesia. The whole body remained active after disinfection with spreading towels in order to ensure the traction and surgical operation. Prepare C-arm X-ray machine and select the TEN of appropriate diameter. The length was required to cover from near metaphysis to far metaphysis. Preflex the TEN, and set the preflexed radian as three times the diameter of the medullary cavity, and the top of its convexity could reach the altitude of the fracture site. The adopted TEN is provided by $\mathrm{AO} /$ ASIF Company and its diameters are $2.5 \mathrm{~mm}, 3.0 \mathrm{~mm}$, $3.5 \mathrm{~mm}, 4.0 \mathrm{~mm}$, and $4.5 \mathrm{~mm}$. Make a small incision of $1.5 \mathrm{~cm}$ near the fracture site of the epiphyseal plate of the wounded limb, blunt dissection to the periosteum which was then incised. At a distance of $2 \mathrm{~cm}$, the mouth 


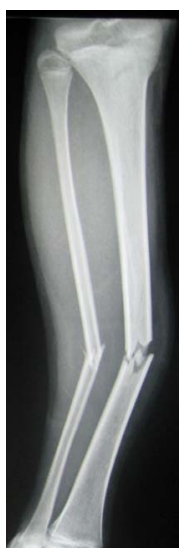

(a)

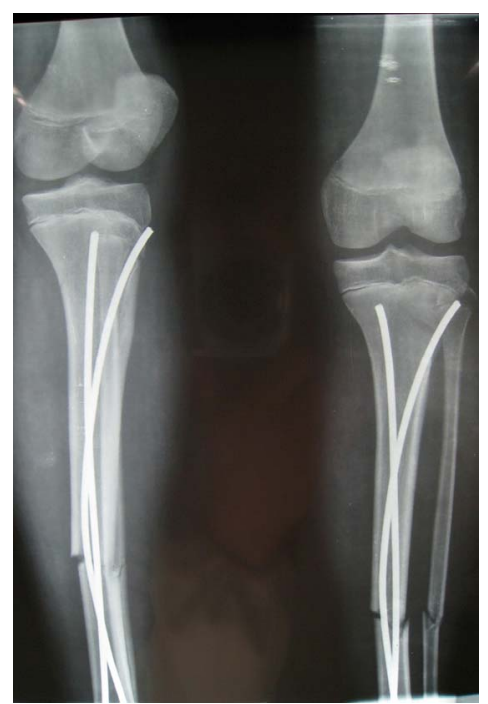

(c)

Figure 1. The X-Ray of tibia fracture of a patient who is 12 years old.

of the mouth-gag was at a 45-degree angle with the diaphysis. Respectively insert two elastic intramedullary nails and push them until the flat of the fracture site, and then keep on inserting the nails after diaplasis until reaching their final positions. Bend the nail tails and lay them next to the bones with the length between 1 - 1.5 $\mathrm{cm}$, then cut the surplus parts. For the cases which were difficult to complete the closed diaplasis, the small incision of the fracture site could be made to assist the diaplasis. In this group of cases, there are twelve cases adopting intramedullary fixation of closed diaplasis. And the other four cases adopt intramedullary fixation by cutting small incisions (Figure 1(c)).

\section{RESULTS}

The operation time is $31-62 \mathrm{~min}$ (48 $\mathrm{min}$ in average), and the amount of bleeding is $60-80 \mathrm{ml}$. The wound heals by Phase I without infection.

The lower tibia silica gel was used to fix the limb for three weeks and during the period, the patients were suggested to perform toes flexion and isometric contraction activity of muscle. After three weeks, the silica gel got removed and the patients performed the flexion activity of ankles and knees.

Fifteen cases were followed up and the follow-up time was 4 - 12 months (4.5 in average). The result: all the fractured patients got healed in Phase I and the average healing time was ten weeks. The near joints' activity and affected extremity completely returned to normal. Two patients showed the symptom of affected extremity shortening (less than $1 \mathrm{~cm}$ ). Eleven patients had their intramedullary nails taken out after six months and no fracture has happened since then. TEN was taken out rapidly without any troubles, and the incision was small.

\section{DISCUSSION}

In recent years, with the development of internal fixation of fracture biology, the intramedullary fixation technology has been highly praised. Doctor Ligier of Nancy hospital in France has been the earliest one to report the clinical outcomes of the operations of the fixation treatment in children's femoral fracture by using TEN. Then TEN operations were popular in Europe and North America and it has become widely used in the treatment of children's fracture of tibia and fibula. ESIN technology not only ensures the protection of epiphysis when treating children's long diaphysis fracture, but also gives consideration to the function diaplasis of fracture treatment. Meanwhile, it does little harm to the tissue, improves the patients' life quality and lessens the complications happening in previous fracture treatment.

The functional principles of stabilizing and treating fracture:

Three-point elastic fixed principle [1]. TEN is preflexed as an arc before being put into use. Then symmetrically insert the TENs from the metaphysic, each nail having three supporting points in the medullary cavity. During the process of inserting, pushing the flexed nails, which are forced to become straight in the medullary cavity, in the relatively straight canal of medullary cavity (compared with the flexed nails). This elastic deformation produces a Bending Moment in the medullary cavity, which promotes the correction of the lateral and angulated displacement of the fracture. When the second intramedullary nail with the same bending angle is inserted into the medullary cavity, it produces the equal and the opposite moment to balance the first nail. Therefore, the cross stress produced by these two 
TENs reaches the goal of maintaining the longitudinal axis of fracture. In the study of biomechanics, this method is called "inside splint" theory [2].

TEN has the following advantages [3]: 1) it belongs to centre-typed inside splint fixation. The mechanical conduction after the fracture fixation is a stress-shared type which has little interference in the limb's biomechanics; 2) the stability of the fracture site is not absolute fixation. When the limb exercises actively or partially bears loads, the fracture site moves slightly, which is good for the formation of poroma and the early healing of fracture; 3) it provides four biomechanical stabilities: anti-flexion stability, axial stability, lateral stability and anti-rotation stability. After the fracture fixation, the fracture can be effectively prevented from displacing, angulating and rotating. TEN is different from Ender and interlocked Kuntscher. Its design of structure makes its operation more suitable for the features of minimally invasive surgery, especially the design of arctyped nail tail makes the inside flexion and passing through the fracture site smoothly of the intramedullary nail when being in closed diaplasis more convenient, and avoids the injury of the epiphyseal plate when inserting and inlaying TEN.

TEN should be used to treat the transverse, shortoblique and short-helical shaft fracture of tibia and fibula. The age should be from 3 - 13 years old and the most suitable age is 5 - 10 years old [4]. The age level should be reduced for obese patients and the range can be widened for those thin children. When treating the short-oblique fracture, especially the large size, the longitudinal displacement cannot be controlled easily. When there is severe comminution and possibility to get the shortening of extremity, the author suggest not using the Elastic nail. TEN's shortage of fixation is lacking of the rotating ability of controlling the fracture site. For the child whose weight is over $50 \mathrm{~kg}$ or whose age is more than 11, some scholars suggests that the TEN should be used with great consideration because of the complications. The author reckons that after the surgery, the focus should be put on the guidance of the patients' recovering exercises. The patients should move their tibiofibulas slightly within a range, which is good for the formation of poroma and the early union of the fracture rather than nonunion. This is very important and it is the factor that many scholars consider the indication has the requirements for age and weight.

When operating TEN, a few issues should be taken into account: 1) choosing the diameter of TEN is vital. The thicker the nail is, the more elasticity it produces, which makes the fixation firmer but the insertion increasingly more difficult. It is reckoned that the size of TEN should be based on the $40 \%$ of the diameter of the narrowest part of medullary cavity [5]. We adopt $2 \mathrm{~mm}$ for children weighing less than $18 \mathrm{~kg}, 2.5 \mathrm{~mm}$ for children weighing between $18 \mathrm{~kg}$ to $28 \mathrm{~kg}, 3 \mathrm{~mm}$ or longer for children with a weight more than $28 \mathrm{~kg}$, and the TENs work well; 2) the intramedullary nail tail is required to lay beside the bone and be flexed slightly, which can help prevent the nail from slipping in the medullary cavity. The tail should only be exposed out of the bone by $1-1.5 \mathrm{~cm}$, otherwise, friction will happen if it is too long, which is called "irritation" phenomenon. Such kind of implication is common and it will cause hematoma and overgrowth of the fracture site [2]; 3) the TEN itself has no function of correcting revolving deformity. Therefore, the correction of the revolving deformity should be paid attention when being in diaplasis. And the revolving deformity after fixation should be prevented from reproducing due to TEN's insufficient ability of anti-rotation; 4) angulated deformity, infection, epiohysis injury, refracture and nail broken, these phenomena happen in low possibility. The author reckons that abundant preparation, choosing appropriate intramedullary nails, careful operation and proper function exercises can keep the mentioned problems away. In terms of the external fixation, the author considers the silica gel is more secured but its using time should be less than 4 weeks. And during this period, the patients should be guided to do some function exercises.

\section{CONCLUSIONS}

ESIN is good for treating the children's long diaphysis fracture which has difficulty in closed diaplasis and is suitable for children aged 6 - 13. ESIN has the advantages of causing small incision, slight injury of soft tissue, short surgery time, rapid recovery and few complications.

\section{REFERENCES}

[1] Cai, Q.X., Chen, T. and Zhang, T. (2007) The application of ESIN in children's diaphisis fracture. Practical Application of Clinical Pediatrics, 22, 317-319.

[2] Flynn, J.M., Hresko, T., Reynolds, R.A. et al. (2001) Tianium elastic nails for pediatric femur fractures. A multicenter study of early results with analysis of complications. Journal of Pediatric Orthopaedics, 21, 4-8.

[3] Hunter, J.B. (2005) The principles of elastic stable intramedullary nailing in children. Injury, 36, Supplement 1, A20-A24.

[4] Gu, Z.P., Yu, X.H., Li, H.Y. et al. (2006) TEN in treating children's fracture of tibia and fibula. Clinical Pediatrics, 5, 98-100.

[5] Flynn, J.M., Skaggs, D.L., Sponseller, P.D., Ganley, T.J., Kay, R.M. and Leitch, K.K. (2002) The operative management of pediatric fractures of the low extremity. Intergalactic Bead and Jewelry Shows, 84, 2288-2301. 